\title{
ON CONVEX SUBSETS OF A POLYTOPE
}

\author{
W. R. HARE, JR. AND C. R. SMITH ${ }^{1}$
}

\begin{abstract}
A. J. Hoffman conjectured the following: Given a $d$-polytope $P$ and a collection, $C_{1}, \cdots, C_{k}$, of closed convex subsets of $P$ with the property that each $t$-flat, $0 \leqq t \leqq d-1$, which meets $P$ also meets some $C_{i}$, then there exist polytopes $D_{j} \subset C_{j}$ such that every $t$-flat which meets $P$ also meets some $D_{j}$. In this note it is shown that the above is true for $k=2$.
\end{abstract}

In [2], Hoffman stated the following conjecture $C(d, t, k)$ : If $P$ is a $d$-polytope, $d \geqq 1$, and $t \geqq 0$ and $k \geqq 1$ are integers, $C_{1}, \cdots, C_{k}$ are closed convex sets in $P$ such that every $t$-flat which meets $P$ meets $\bigcup_{i=1}^{k} C_{i}$, then there are polytopes $D_{1}, \cdots, D_{k}$ with $D_{i} \subset C_{i}, 1 \leqq i \leqq k$, such that every $t$-flat which meets $P$ meets $\bigcup_{i=1}^{k} D_{i}$ also. He established $C(d, 0, k)$ in this same paper. Zaks ([3], [4], [5]) has shown that $C(d, d-2, k), d \geqq 3$, $k \geqq 4$, is false, that each of $C(d, d-1, k)$ for all $d$ and $k, C(d, t, 1)$ and $C(3,1,3)$ is true.

The purpose of this note is to prove $C(d, t, 2)$ for which two lemmas are established and the resulting theorem follows. (For notation, see Grünbaum [1].)

Lemma 1. Let $P$ be a d-polytope and $C_{1}, \cdots, C_{k}$ be closed convex subsets of $P$ such that every t-flat that meets $P$ meets $\bigcup_{i=1}^{k} C_{i}, 0 \leqq t \leqq d-2$; then skel ${ }_{m} P \subset \bigcup_{i=1}^{k} C_{i}$, where $m=d-t-1$.

Proof. Let $F$ be an $m$-face of $P$, and let $H$ be a supporting hyperplane of $P$ such that $H \cap P=F$; let $x$ be an arbitrary point of $F$. The affine flat in $H$, orthogonal to the affine hull of $F$ and passing through $x$, is of dimension $d-1-m=d-1-(d-t-1)=t$; it meets $P$ in exactly $\{x\}$; therefore by the assumption on $C_{1}, \cdots, C_{k}$, we have $x \in \bigcup_{i=1}^{k} C_{i}$ as promised.

Lemma 2. Suppose $P$ is a d-polytope and $C_{1}$ and $C_{2}$ are closed convex subsets of $P$ such that skel ${ }_{1} P \subset C_{1} \cup C_{2}$; then $P=C_{1} \cup C_{2}$.

Proof. If $P \neq C_{1} \cup C_{2}$, then there is a face $F$ of lowest dimension $m \geqq 2$ such that $F \leftarrow C_{1} \cup C_{2}$. By minimality of $m$, bd $(F) \subset C_{1} \cup C_{2}$.

Received by the editors May 19, 1971 and, in revised form, November 18, 1971. AMS 1969 subject c'lassifications. Primary 5210, 5230; Secondary 5090.

Key words and phrases. Convex polytope, closed convex set, affine flat, convex hull. ${ }^{1}$ Latter author supported by NSF Fellowship No. 60175. 
Let $p \in \operatorname{rel} \operatorname{int}(F) \backslash\left(C_{1} \cup C_{2}\right)$ and consider the mapping $x \rightarrow x^{\prime}$ defined as follows. For $x \in \operatorname{bd}(F)$, let $x^{\prime}$ be the (unique) point of $\operatorname{bd}(F)$ such that the line $L(p, x)$ meets $F$ in the segment $x x^{\prime}$. By the choice of $p$, it follows that $x$ and $x^{\prime}$ are in distinct members of $\left\{C_{1}, C_{2}\right\}$. Now

$$
\operatorname{bd}(F)=\left(\operatorname{bd}(F) \cap C_{1}\right) \cup\left(\operatorname{bd}(F) \cap C_{2}\right)
$$

and $\left(\operatorname{bd}(F) \cap C_{1}\right) \cap\left(\operatorname{bd}(F) \cap C_{2}\right)=\varnothing \quad$ with $\quad \operatorname{bd}(F) \cap C_{1}$ and $\operatorname{bd}(F) \cap C_{2}$ nonempty. Since $C_{1}$ and $C_{2}$ are closed, this contradicts the connectedness of $\operatorname{bd}(F)$.

Theorem. Let $P$ be a d-polytope and let $C_{1}$ and $C_{2}$ be closed convex subsets of $P$ such that every $t$-flat, $0 \leqq t \leqq d-1$, which meets $P$ also meets $C_{1} \cup C_{2}$. Then there exists polytopes $D_{1} \subset C_{1}, D_{2} \subset C_{2}$ such that every $t$-flat which meets $P$ meets $D_{1} \cup D_{2}$.

Proof. From the lemmas, $P=C_{1} \cup C_{2}$; hence Hoffman's theorem in [2] is sufficient to complete the proof.

The portions of $C(d, t, k)$ which remain open are for $0<t \leqq d-3$ with $d \geqq 4$ and $k \geqq 3$.

\section{REFERENCES}

1. B. Grünbaum, Convex polytopes, Pure and Appl. Math., vol. 16, Interscience, New York, 1967. MR 37 \#2085.

2. A. J. Hoffman, On covering of polyhedra by polyhedra, Proc. Amer. Math. Soc. 23 (1969), 123-126. MR 40 \#835.

3. J. Zaks, On a conjecture of A. J. Hoffman, Proc. Amer. Math. Soc. 27 (1971), 122125.

4. - On a conjecture of A. J. Hoffman. II, Proc. Amer. Math. Soc. (to appear).

5. - On Hoffman's conjecture (manuscript).

Department of Mathematics, Clemson University, Clemson, South Carolina 29631

Department of Mathematics, Northeast louisiana University, Monroe, LOUISIANA 71201

Department of Mathematics, University of Washington, Seattle, WashingTON 98105 